\title{
Mass spectrometry in high throughput analysis
}

\author{
Elizabeth Want ${ }^{\mathrm{a}, \mathrm{b}}$, Michael Greig ${ }^{\mathrm{c}}$, Bruce Compton ${ }^{\mathrm{d}}$, Ben Bolaños ${ }^{\mathrm{c}}$ and Gary Siuzdak ${ }^{\mathrm{a}, \mathrm{b}, *}$ \\ ${ }^{a}$ The Scripps Research Institute, 10550 North Torrey Pines Road, La Jolla, CA 92037, USA \\ ${ }^{\mathrm{b}}$ Mass Consortium Corporation, 3030 Bunker Hill Street, San Diego, CA 92109, USA \\ ${ }^{\mathrm{c}}$ Pfizer Global Research and Development - La Jolla Laboratories, Agouron Pharmaceuticals, Inc., \\ 10770 Science Center Drive, San Diego, CA 92121, USA \\ ${ }^{\mathrm{d}}$ Waters Corporation, 34 Maple Street, Milford, MA 01757, USA
}

\section{Overview}

High throughput mass spectrometry has been motivated largely from recent developments in both chemistry and biology. For instance, in chemistry, the production of large populations of molecular libraries increases the probability that novel compounds of practical value will be found, yet also requires that their identity be confirmed and purity assessed [1-6]. While many fields of research have been influenced by this approach, the largest investment has come from the pharmaceutical, biotechnology and agrochemical arena. In the field of drug development, high throughput chemistry represents a convergence of chemistry with biology, made possible by fundamental advances in automation, such as that of mass spectrometry [7-19]. High throughput technology has even been extended to proteomics, where mass spectrometry is being used as a tool in rapid protein identification [20-22].

Electrospray ionization (ESI), atmospheric pressure chemical ionization (APCI), and matrix-assisted laser desorption/ionization (MALDI) mass spectrometry have become useful for qualitative, and more recently, quantitative analysis, aiding the development of mass spectrometry as a high throughput tool in these different fields (Fig. 1) [2-4,9-16]. Moreover, the advancement of these techniques has significantly extended MS applications toward a wide variety of challenging problems in drug discovery and also toward the identification of effective new catalysts and enzyme inhibitors $[3,9,10]$. In addition, because MS based methods do not involve chromophores or radiolabelling, they provide a viable alternative to existing analytical techniques which typically require extensive sample preparation and optimization time, the disposal of biohazardous waste, or a significant amount of sample.

The utility of ESI lies in its ability to generate ions directly from the liquid phase into the gas phase, establishing this technique as a convenient mass detector for both liquid chromatography and automated sample analysis. In addition, ESI-MS offers many advantages over other mass spectrometric ionization techniques, including the ability to analyze low and high mass compounds, excellent quantitation and reproducibility, high sensitivity, simple sample preparation, amenability to automation, soft ionization, and the absence of matrix (as is necessary for MALDI). APCI, much like ESI, generates ions from a liquid stream, but is a somewhat harsher ionization technique than ESI. Because APCI imparts more energy

\footnotetext{
*Corresponding author. E-mail: siuzdak@scripps.edu.
} 


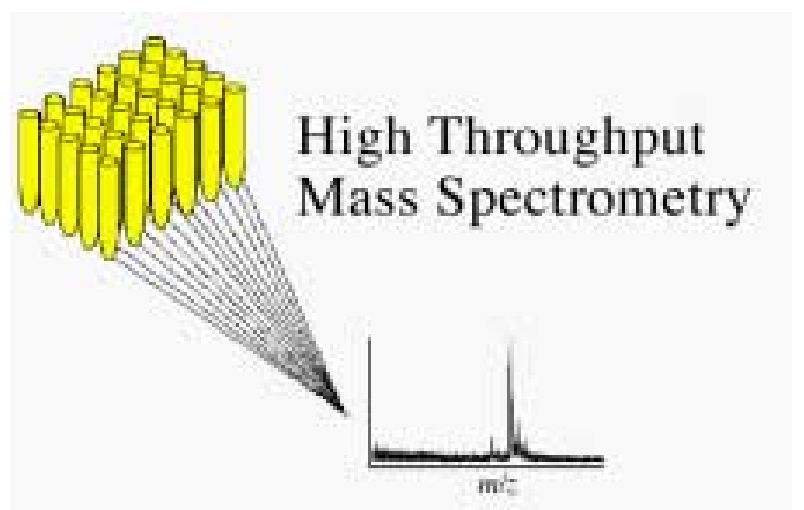

Fig. 1. Mass spectrometry in high throughput analysis involves the analyses of hundreds or thousands of compounds using atmospheric pressure ionization (API) liquid chromatography/mass spectrometry (LC/MS) or surface based laser desorption/ionization (LDI) approaches such as matrix-assisted laser desorption/ionization (MALDI) or desorption/ionization on silicon (DIOS) mass spectrometry.

into molecules for ionization than ESI, it is able to generate ions from less polar molecules than typically seen with ESI, but generally increases background signal along with that of the ion of interest. Fragmentation may occasionally occur using APCI, but the molecular ion is often still the most dominant. APCI is limited in its applicable mass range $(<1000$ Daltons) due to its inherent nature. A third atmospheric source, atmospheric pressure photoionization (APPI) has also begun to appear as a useful ionization tool [23-25]. This source uses photons emitted from a krypton discharge lamp (10.0 and $10.6 \mathrm{eV})$ to ionize molecules, and has been shown to be particularly effective for the analysis of steroids - molecules difficult or impossible to detect using other atmospheric pressure ionization techniques. MALDI-MS generates gas phase ions by the laser vaporization of a solid matrix/analyte mixture. MALDI is especially well suited for the simultaneous analysis of multicomponent mixtures and commercial instruments are now available that offer automation capabilities. Finally, DIOS generates gas phase ions by the direct laser vaporization of an analyte on a silicon surface. Since DIOS does not require a matrix, it does not create low mass interference and can therefore be used for small molecule analysis. In the following sections, the practical aspects of automated mass analysis are covered and also how the unique ionization properties of ESI, APCI, APPI, MALDI and DIOS have allowed mass spectrometry to become a powerful tool in high throughput analysis.

\section{Automating atmospheric pressure ionization sources (ESI, APCI, \& APPI)}

\subsection{Sample preparation/instrument configuration}

"One bad apple does ruin the whole bunch" Mike Greig (Pfizer)

The basic elements in successful mass analysis include sample solubility, sample purity, instrument calibration and sensitivity (is there enough sample present?). In automated mass spectrometry, where hundreds or thousands of analyses are performed each day, these elements become even more important. Solubility is a significant consideration, because if a sample turns into a suspension then the particulates can damage the MS system by clogging lines and autoinjectors. Filtering samples prior to their introduction into the MS system is one answer to this problem. Issues such as instrument calibration and 
sensitivity are primarily a matter of instrument maintenance and can be routinely monitored by incorporating standards at planned intervals.

Additional sample considerations in regards to automated analysis are: carryover, salt contamination, labeling, and sample introduction. Carryover is just as detrimental to mass analyses as clogging. Samples that easily ionize and are in the solution at high concentration are susceptible to carryover signal for subsequent analysis performed. The solution is simple, but adhering to minimized concentrations can sometimes be difficult because the individual researcher may not always know exactly how much material is present. One approach to overcome this problem is to randomly monitor the UV absorption at the beginning of a large run, as high absorbance measurements of the initial samples can be used as criteria to shut down the system, send out a warning, or in more advanced systems, divert the flow to waste before the material reaches the mass spectrometer. Concerning salt contamination, some instruments can be surprisingly tolerant of high salt levels in solutions. Fortunately, the problem of salt inhibiting electrospray ionization can be easily avoided by several methods, including Zip-tipping the sample or performing LC/MS. In regards to labeling, it is best to institute a labeling system prior to beginning a large number of samples. While this may seem obvious, the value of organizing and putting a little thought into the labeling scheme can be very valuable.

Another relevant question that is frequently being asked in MS laboratories is whether to perform flow injection or LC/MS. While LC/MS has become more rapid, often taking less than 5 minutes per run, flow injection is faster, typically requiring less than a couple minutes per analysis (and in some cases less than one minute). Also, with flow injection all the information can be obtained in one spectrum. The primary disadvantage is that significant signal suppression can occur when a complex mixture is introduced, thus resulting in a loss of information and possibly a dip in sensitivity. This is where LC/MS becomes especially advantageous with its ability to separate out the mixture components; but two disadvantages are the reduction in the speed of the analysis and the additional time required to analyze the data.

The type of sample being analyzed is yet another consideration with automated API analysis. Typically the analysis of large molecules requires parameter manipulation, such as that of the fragmentor voltage (also known as orifice potential) to obtain a reasonably good signal. Small molecule analyses will often give good ion signals (typical ions observed are shown in Table 1) under a large variety of conditions and therefore are easier to automate.

Further practical considerations for automated sample analysis include regulating flow rates, minimizing waste generation, venting, pump oil maintenance, solvent quality, safety and comfort. Lower flow rates allow the use of less solvent, which in turn generates less waste and minimizes some venting concerns. Of course, performing thousands of analyses at high flow rates requires consistent system maintenance including ionization source cleaning and regularly scheduled pump oil change, as pump oil breakdown can lead to many problems. Using high quality GC grade solvents (as opposed to LC grade which are only UV pure) is also advantageous for minimizing background ions which may be detected by MS. Importantly, safety and comfort around the instrument should be established as early as possible.

Table 1

Ions typically monitored for automated mass analysis with screening performed in both positive and negative ionization modes

\begin{tabular}{ll}
\hline Positive ionization mode & Negative ionization mode \\
\hline$[\mathrm{M}+\mathrm{H}]^{+}$ & {$[\mathrm{M}-\mathrm{H}]^{-}$} \\
{$[\mathrm{M}+\mathrm{Na}]^{+}$} & {$[\mathrm{M}+\mathrm{Cl}]^{-}$} \\
{$[\mathrm{M}+\mathrm{K}]^{+}$} & {$[\mathrm{M}+\mathrm{TFA}]^{-}$} \\
\hline
\end{tabular}


Lastly, be sure to provide adequate space and ventilation around the instrument in order to access the system for inevitable problems that arise with all mass spectrometers.

\subsection{Data analysis/software/storage}

The ability to analyze the data from the mass spectrometer should be a major consideration when developing an automated mass spectrometry analysis system. While the instrumentation is becoming more and more reliable, the MS system software is constantly evolving. Therefore, it is becoming more important to define the problem and/or specific needs before searching for the system that will answer them. Whether it is to be used for routine batch analysis, high-throughput pharmokinetic studies, or a search for new inhibitors, the software programs associated with each of the manufacturers' different mass spectrometers will allow these problems to be tackled in different ways, some faster and better than others.

Data storage can also become an issue when generating large or numerous data files. An average MS data file requires anywhere from $25 \mathrm{~Kb}$ to $250 \mathrm{~Kb}$ of memory storage, so even the large hard drives in modern PCs can be overwhelmed rapidly. Currently, the more expensive magnetic based storage drives are fast, easy to use and rewritable. However the low cost CD-based storage mediums are also attractive, so a decision can be made depending on the cost (CD) to "ease of use" ratio (magnetic) as to what is most important. The writing, transferring, and eventual deletion of massive amounts of data quickly leads to hard drives that can be fragmented beyond repair. Consequently, it is important to defragment hard drives on a regular basis using any of a number of commercially available software packages.

\section{Typical equipment being used for automated API analyses}

API-MS systems may be configured with various mass spectrometers, each of which has its benefits and limitations. The quadrupole is by far the most commonly used mass analyzer because of its low cost, manageable size and robustness for coupling to liquid chromatographs. A quadrupole can provide adequate mass resolution and mass accuracy, but it is not the MS of choice for applications that require high performance measurements (Table 2). More importantly, the primary limitation of the quadrupole is its low sensitivity when detecting across a broad mass range, which may be a serious consideration when the mass of interest is not known in advance. As an alternative, both the quadrupole ion trap and the

Table 2

General comparison of ESI mass analyzers. It should be noted that performance can vary significantly for different commercial systems

\begin{tabular}{|c|c|c|c|c|c|}
\hline & Accuracy & Resolution & $m / z$ Range & Tandem MS & Scan speed \\
\hline Quadrupole & $0.01 \%$ & 5000 & 4000 & $\mathrm{MS}^{2}$ & seconds \\
\hline Comments: & \multicolumn{5}{|c|}{$\begin{array}{l}\text { Large dynamic signal range, small size, relatively low cost, - has proven to be robust in combinatorial } \\
\text { library analysis. }\end{array}$} \\
\hline Ion trap & $0.01 \%$ & 5000 & 4000 & $\mathrm{MS}^{4}$ & seconds \\
\hline Comments: & \multicolumn{5}{|c|}{$\begin{array}{l}\text { Limited dynamic signal range, small size, relatively low cost, well suited for tandem mass spectrometry, } \\
\text { - somewhat useful for combinatorial library analysis. }\end{array}$} \\
\hline TOF-reflectron & $\begin{array}{l}0.003 \% \\
\text { or better }\end{array}$ & 10,000 & 10,000 & $\mathrm{MS}^{2}$ & milliseconds or better \\
\hline Comments: & Very fast s & $\mathrm{d}$, simple d & ood resolving & & \\
\hline
\end{tabular}


time-of-flight (TOF) mass spectrometers are viable analyzer options which address some of the inherent limitations of the quadrupole. Ion traps provide the unique means to perform multiple stages of MS for fragment identification, or ion-molecule reactions, with improved mass resolution over that achievable by quadrupole-MS. However, the ion trap still requires slow scanning for high mass resolution and may not be ideally suited for high speed analysis. In the movement towards high throughput-analysis, TOF systems have become increasing popular because they tackle many of the limitations of the previous two MS instruments. As a pulsed detector, TOF scan speeds are exceedingly fast, on the order of milliseconds. This pulsed detection does not discriminate against ion mass and so the $m / z$ range is infinite, a quality unique to TOF analyzers. Lastly, with improved electronics incorporated into today's TOF systems, mass accuracy has been significantly enhanced and TOF MS is used routinely for mass accuracy measurements $(<5 \mathrm{ppm})$, where mass formula validation or identification may be used.

\section{Automated quantitative ESI-MS as an assay for enzyme inhibitors}

Using the advantages of ESI-MS detailed, one specific application of automated ESI-MS is the investigation of inhibitor libraries designed to inhibit enzymatic glycosylation reactions [9]. In this approach, the entire enzymatic mixture (substrate, inhibitor, product, and internal standard) is introduced into the ESI mass spectrometer while analyzing for product formation as a function of the presence of inhibitor. Since only the product formation is quantitatively monitored, the effectiveness of the inhibitor can be readily determined.

As an example, we have applied this automated quantitative ESI-MS approach (Fig. 2) to monitor inhibitors of galactosyltransferase [10] and fucosidase enzymes [9]. Both enzymes are responsible for processing cell-surface carbohydrates, which in turn are associated with many specific recognition and signaling processes leading to important biological functions and disease. Therefore, developing new

\section{Monitoring Enzyme Inhibition with Mass Spectometry}

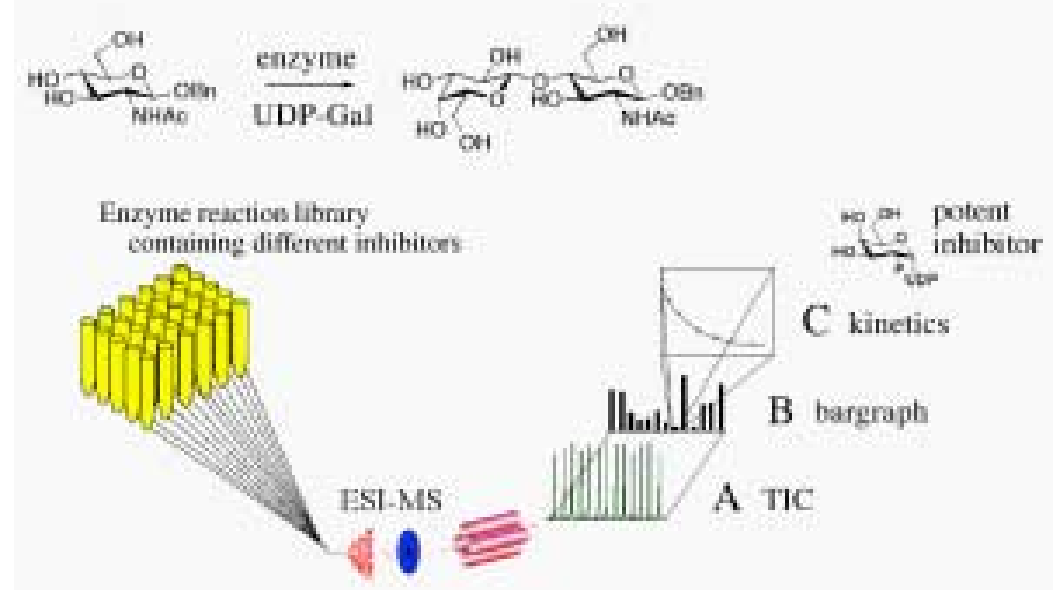

Fig. 2. An enzyme reaction was monitored by an automated electrospray ionization mass spectrometry screening experiment for quantitative determination of galactosyltransferase inhibition. Each solution contains the enzyme, reactants, an inhibitor (inset), and an internal standard. (A) The total ion current (TIC) is recorded on each injection into the mass spectrometer. (B) Product formation is then monitored with respect to the internal standard during each injection as a bargraph, and (C) if an inhibitor is found to be effective, $\mathrm{IC}_{50}$ data can be generated using ESI-MS. 
assays and finding new inhibitors for glycoprocessing reactions is both medically relevant as well as scientifically interesting. To date however, only limited success has been achieved in the design of these inhibitors. Current assays for the quantitation of glycosyltransferase activity require the separation of the compounds, the use of radiolabeled sugars, the performance of large scale reactions, or the use of additional enzymes.

\subsection{Experimental example of screening enzyme inhibition}

In the first set of experiments, a galactosyltransferase catalyzed reaction was examined in the presence of potential inhibitors [10]. The 20 inhibitor candidate and 2 control reactions of the initial inhibitor library were assayed individually in 22 parallel reactions (Fig. 3), and then quenched by adding $\mathrm{MeOH}$

Mass spectral data from each injection

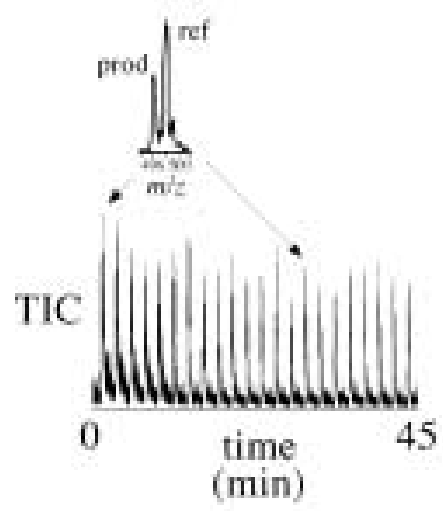

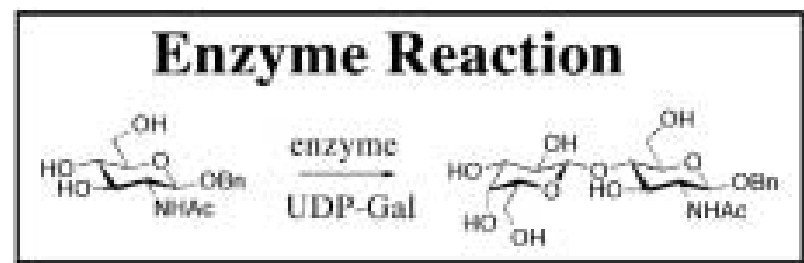

Bargraph representing inhibitors relative activity
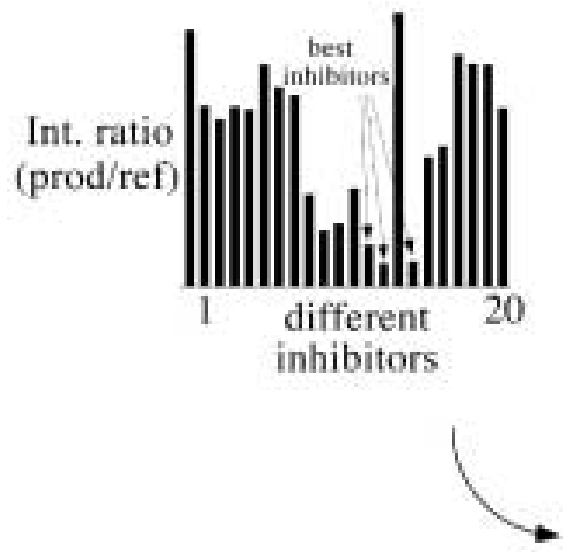

Quantitative data on an inhibitor's activity against the enzyme

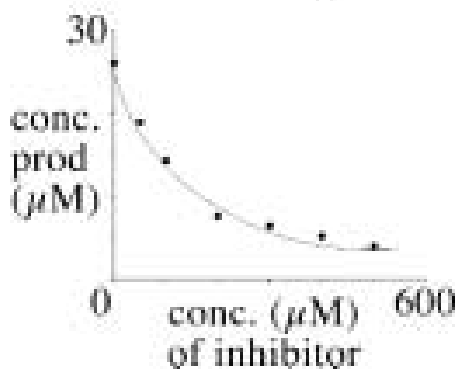

Fig. 3. (Left) Electrospray ionization total ion current observed for the 22 reactions (each having a different inhibitor). Insets are the mass spectrum for the reaction with no inhibitor. (Middle) The bargraph of the ratio product/reference generated from the mass spectrum of each reaction indicated which inhibitors were active. (Right) Quantitative data on the product of the galactosyltransferase catalyzed enzyme reaction was also monitored as a function of inhibitor concentration to generate the $\mathrm{IC}_{50}$ data. 
(followed by the addition of an internal standard). The reaction mixtures were then injected directly into the electrospray mass spectrometer with an autosampler. The concentrations of the inhibitors were varied in order to determine the $\mathrm{IC}_{50}$ (inhibitor concentration at $50 \%$ inhibition).

By monitoring one inhibitor at a time, we could examine the effectiveness of a new inhibitor every two minutes. In an effort to further increase the screening capacity, multiple inhibitors were screened against one enzyme simultaneously. The experiments were performed by pooling 5 potential inhibitors and the enzyme into one reaction vessel. The reactions that contained 5 inactive compounds showed no inhibition, whereas the reactions containing 4 inactive compounds and 1 active inhibitor (UDP) showed potent inhibition. The compounds in the inhibited reaction were then analyzed individually to identify the actual inhibitor compound. Such strategies increased the screening capacity five fold such that 1 inhibitor/2 min allowed for 720 inhibitors/day, while 5 inhibitors/2 min allowed for 3600 inhibitors/day.

While we have shown the effectiveness of this method in the screening of inhibitors for enzymes involved in carbohydrate processing, one can envision how this approach can be extended toward screening libraries of other classes of enzymes (such as enzymes with peptide-based substrates) or metal-catalyzed chemical reactions. It is important to note that the only criterion of this approach is that the substrate or product molecule is ionizable.

\section{1 Million analyses/year: A case study}

The task at hand was to analyze one million discrete compounds derived from high throughput chemistry in one year using an MS based technique. If one were to assume the syntheses yielded pure or at the least very clean reactions, the obvious choices would be to use either a rapid flow injection technique with any number of different types of instruments, or DIOS coupled with rapid sample preparation. In reality, where reaction yields can be anywhere from 10-90\%, a chromatographic based MS technique will produce the best results when estimating purity. The three choices reviewed here are multiple LC/MS systems using a durable single quadrupole mass spectrometer, multiple LC systems flowing into a single MUX, or multiplexed, MS, or ultra-high speed super critical fluid chromatography (SFC) with a time of flight (TOF) MS. Each system can analyze about one million compounds per year, with their own advantages and disadvantages.

The simplest and most dependable method of achieving this throughput goal would be to use eight separate LC/MS systems. This option provides the most flexibility, as any combination of LC or mass spectrometer brands and types can be put into service with any combination of API sources. Additionally, should one system break down, there would be seven remaining systems continuing to produce data. Since modern LC/MS systems are quite robust, inexperienced operators can still obtain quality data. Assuming a 3 minute cycle time (time from injection to injection) on a system running an average of five days a week for 24 hours each day, eight systems could analyze 992,000 samples in one year. Although a seemingly simple solution, it would take considerable man power (to load instruments, analyze data, and perform preventative maintenance) to keep these systems operational. Additionally, data would be produced on eight different instruments, and the required laboratory space and capital investment for these systems is quite large.

A second option would be to use a single 8x MUX MS system fed by eight HPLCs. For ESI only systems, a MUX, or multiplexing system, is currently one of the more advanced for rapidly sampling from numerous HPLCs with one mass spectrometer (Fig. 4) [26]. The concept is simple in that multiple ESI sources are placed around the entrance to a TOF mass analyzer, then each source is sampled by 


\section{Liquid Chromatography ESI MUX mass spectrometry}
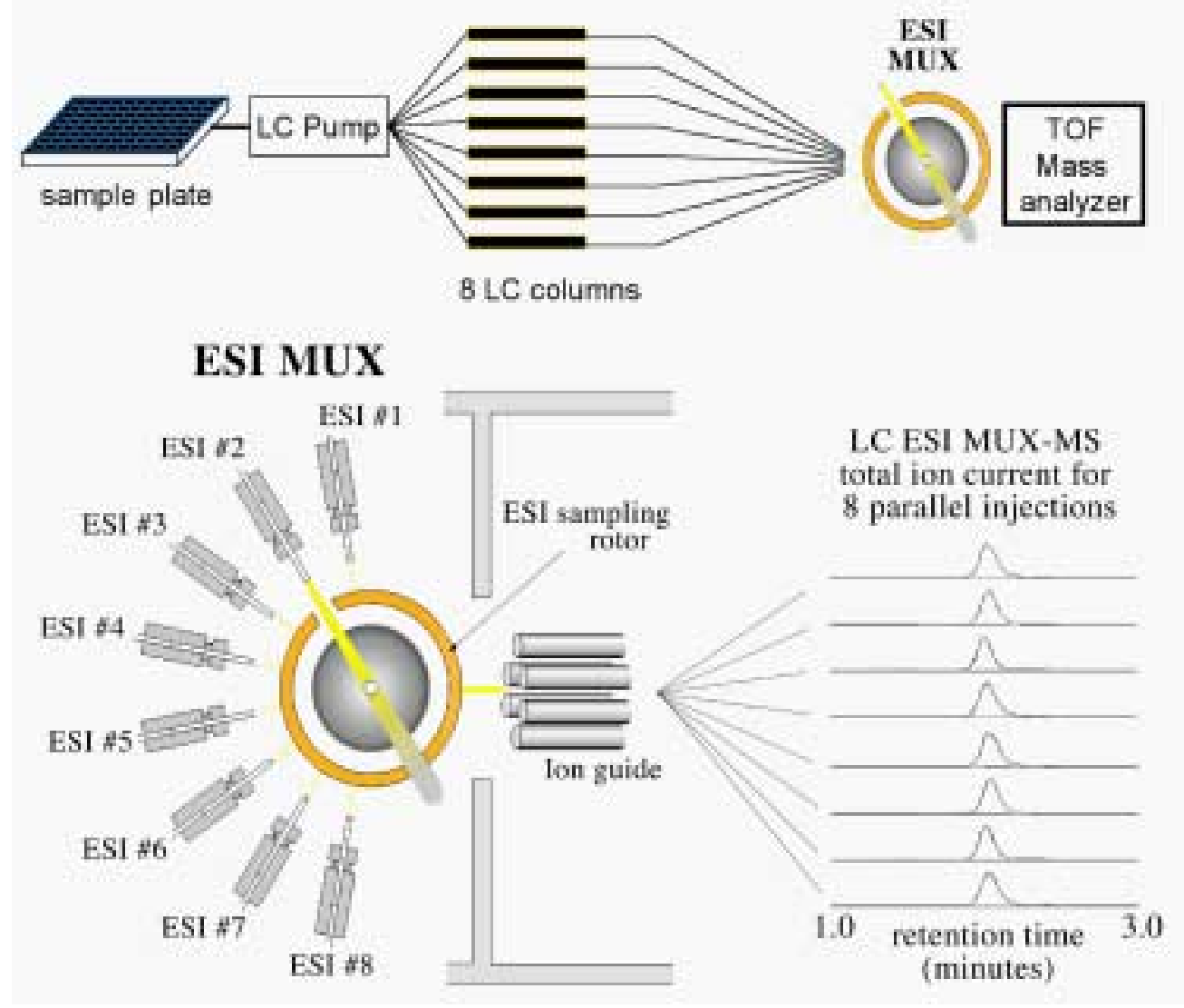

Fig. 4. Multiplexing multiple ESI sources onto a single mass spectrometer is one way of increasing sample throughput. This example shows eight ESI heads going into one time-of-flight mass analyzer. The mass analyzer samples each ESI head via a rotating rotor that rapidly moves from one ESI port to another.

moving a rotor that allows only one ESI source to spray into the mass analyzer at a time. The advantages of this setup are that as many as eight different LC systems can be analyzed in parallel, one data set is acquired using a single data station, it needs relatively little bench space, and one skilled operator can keep the system operational. The disadvantages are that while this wheel is spinning, each channel is sampled less than $1 / 8$ of the time, resulting in fewer data points across each peak. Because of this, slower LC methods must be employed, resulting in a "fast" run with a cycle time of five minutes. Using the slower LC method, this system could analyze about 750,000 compounds/year running in the same time frame as previously described. The second and major disadvantage is if the system or any single component is inoperative, there is no back-up system and no data will be produced until the entire system is repaired.

A third, lesser known option, is to couple supercritical fluid chromatography (SFC) with an API-TOF MS. SFC is rapidly gaining popularity in the pharmaceutical industry due to its high speed and applicability to most drug-like chemicals $[6,19,27]$. SFC is a normal phase chromatographic technique that uses compressed $\mathrm{CO}_{2}$ as the mobile phase which is then modified by a polar solvent such as methanol. Be- 


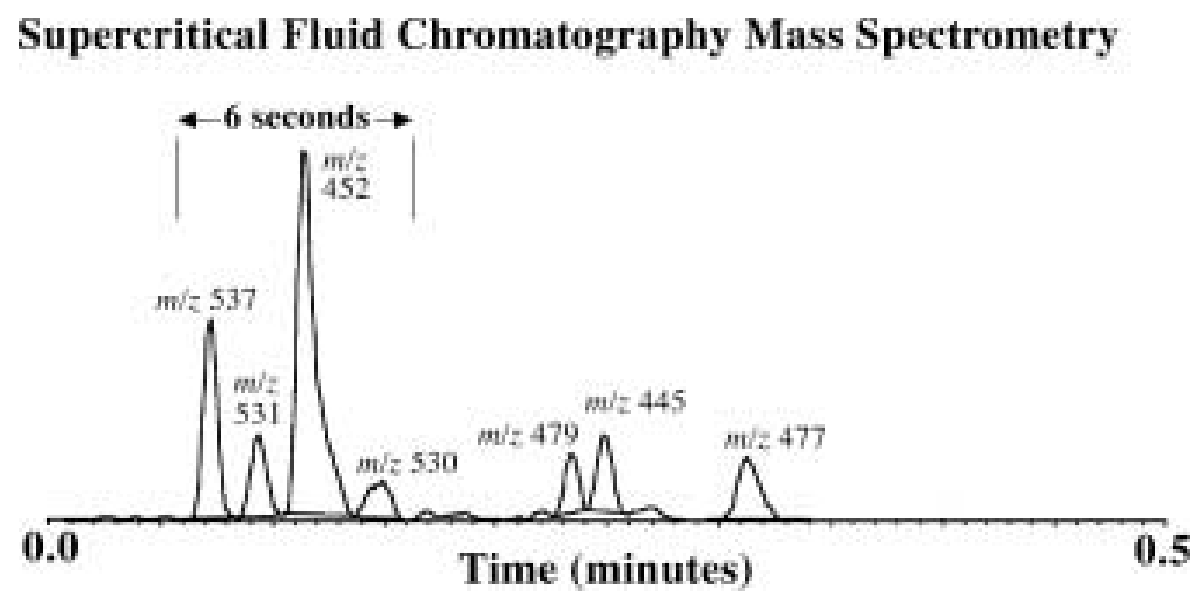

Fig. 5. Analysis of a complex mixture by the ultra-fast SFC method. SFC uses compressed $\mathrm{CO}_{2}$ as the mobile phase which is then modified by a polar solvent such as methanol to generate a gradient. Measurement of baseline separation of four eluting compounds within a window of 6 seconds is also facilitated by the fast $0.05 \mathrm{sec} / \mathrm{scan}$ data point sampling using an APCI-TOF-MS.

cause of the higher diffusivity and lower viscosities achieved by the mobile phase, SFC can be run at much higher flow rates than standard LC, with higher resolution. Running at $10 \mathrm{ml} / \mathrm{min}$ with a methanol gradient from 5-60\% over thirty seconds, a forty second cycle time was achieved while still providing good resolution (Fig. 5) [28]. At this rate, with two instruments, 1.2 million samples per year could be analyzed. This system has several advantages over the previous two described above. Since there are duplicate systems, one system breaking down will not mean a complete loss of data. Only one or two skilled operators are needed, and the capital outlay is much lower than the previous options. Another major advantage comes in solvent usage and waste production. Both the MUX and multiple LC/MS systems require eight HPLC systems, each consuming a considerable amount of organic solvent. Two SFC systems, while at a much higher flow rate, consume about $17 \%$ of the organic solvent. ESI, APCI, and APPI have all been demonstrated to work well with SFC, so unlike a MUX system, there is a choice of API source. The major disadvantage of an SFC/MS system is that there are very few commercially supported systems available and few operators experienced with both SFC and MS.

In the examples discussed, API-MS was the mass spectrometric method of choice mainly because of its ability to analyze low mass compounds and its amenability to coupling with a chromatographic system. As the following section will show, MALDI-MS, although requiring the use of a matrix, or DIOS, which requires no matrix, offer many of the same advantages (automation, soft ionization, and excellent quantitation) and in addition are able to analyze high mass compounds and relatively heterogeneous mixtures.

\section{Automating MALDI and DIOS-MS}

One bad apple doesn't have to ruin the whole bunch

Two of MALDI's and DIOS' greatest attributes are their ability to analyze complex heterogeneous mixtures and to scan many samples rapidly. This has made them valuable tools in the analysis of biological fluids, commonly performed by immunoassays, high performance liquid chromatography (HPLC) 
and HPLC/ESI-MS. Immunoassays, however, generally have low reproducibility and reliability, and provide little or no selectivity between a drug and its metabolites. This lack of specificity is a significant limitation, since metabolites, although structurally similar to the parent compound, often have different biological activity. In addition, both HPLC and HPLC/ESI-MS are relatively selective and accurate; the sensitivity of HPLC is very compound-dependent and method development and run times for both HPLC and HPLC/ESI-MS can be time-consuming. MALDI and DIOS mass spectrometric analysis, on the other hand, allows for co-extracted metabolites to be identified and quantitatively monitored (unless they have the same molecular weight). Therefore, there are tremendous benefits that can be gained from automating the MALDI analysis procedure.

\section{Pattern searching}

The automation of MALDI and DIOS analyses is becoming increasingly important in proteomics and combinatorial chemistry [5,11,20-22]. Typically, the instrument is designed to search for a signal from each sample well. Analyses are driven by a computer-controlled procedure that monitors for the ion signal as a function of laser position and laser intensity. To accomplish this, the computer workstation automatically adjusts laser intensity and searches the sample well until a signal (within the specified mass range and intensity threshold) is obtained (Fig. 6). Based on a careful preselection of autosampler options from manual analysis of the sample, each parameter (laser intensity, search pattern and step size in well, signal within a specified $m / z$ range, and $m / z$ range) is adjusted to minimize time of analysis and maximize signal quality.

In an example of work performed in our laboratory, what follows is a brief description of a MALDI and DIOS automation procedure (Figs 7 and 8). The laser intensity was initially at a minimum energy setting of $\sim 2 \mu \mathrm{J} /$ pulse and was then increased up to a maximum of $50 \mu \mathrm{J} /$ pulse (as controlled by a variable neutral density filter) in step sizes of five increments, resulting in an increase in laser intensity of

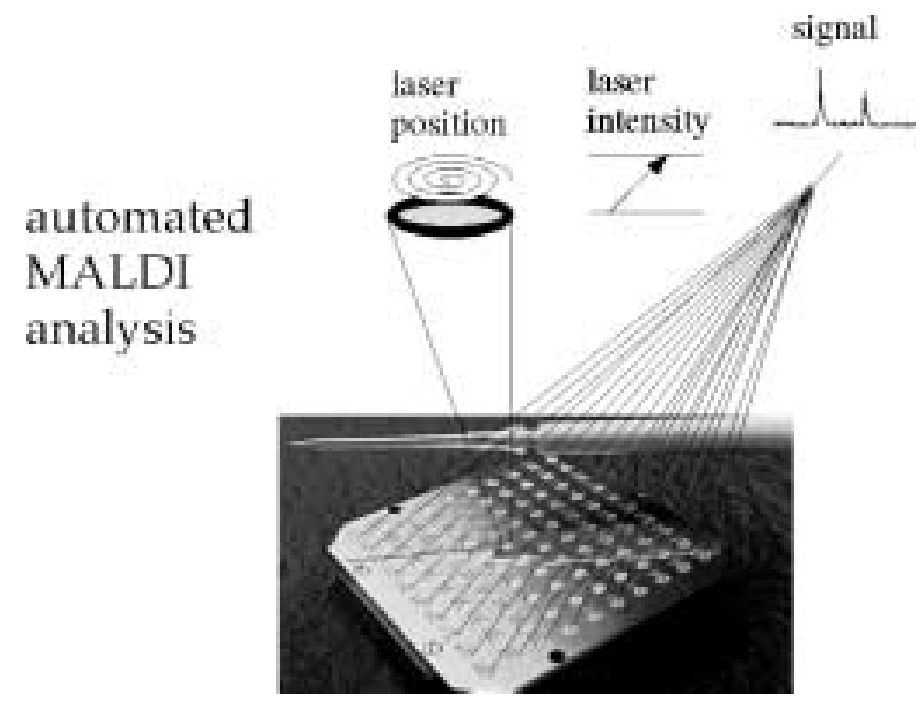

Fig. 6. Three different parameters to adjust and monitor using the autosampler mode on a laser desorption/ionization system: (1) automatically adjust laser position, (2) automatically adjust laser intensity, (3) specify acceptable mass \& intensity range for the signal. 


\section{DIOS Silicon Chip}

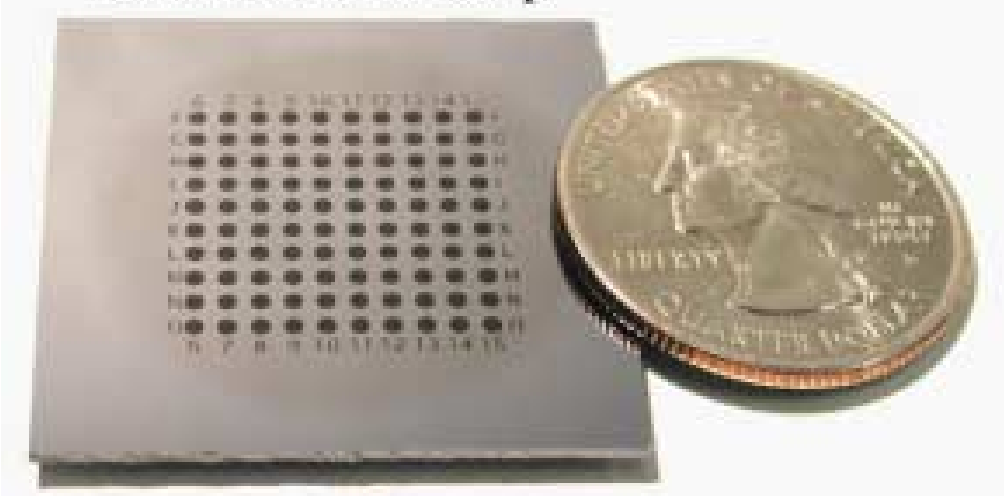

Fig. 7. Desorption/Ionization on Silicon (DIOS) mass spectrometry chip used for high throughput mass spectrometry experiments.

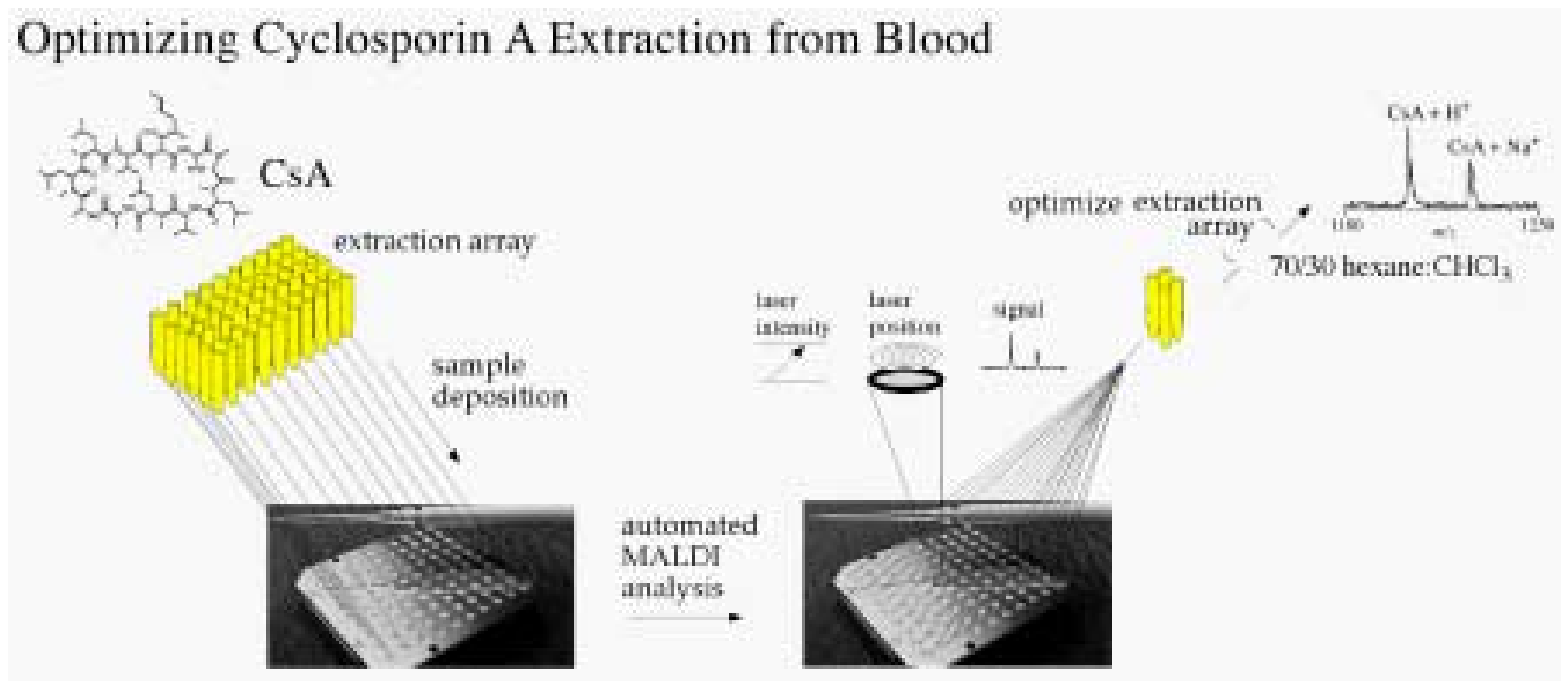

Fig. 8. Automated MALDI mass spectrometry experiments, performed as a function of laser position (within each well) and laser intensity for each extracted CsA sample. The first iteration of these extraction experiments generated four useful solvent systems, and the second generation of these experiments produced an effective $70: 30$ hexane/ $\mathrm{CHCl}_{3}$ extraction solvent system.

approximately $10 \mu \mathrm{J} /$ pulse per step. The laser intensity was increased until an acceptable data signal was acquired, whereby if no signal was observed, the laser beam was repositioned on the well and the analysis resumed at the lower laser power. To adjust the laser position on the sample plate, a pre-programmed spiral search pattern was used which began in the center of each circular well and spiraled outward in $0.2 \mathrm{~mm}$ increments. For each sample well analysis, only signals that reached a specified intensity were saved and once this signal was observed the analyses would automatically move to the next well. On average, the total time spent for each sample well analysis was 140 seconds. This included acquisition time for averaging 64 scans and a delay for adjusting laser intensity and repositioning the sample plate. 
The careful selection of the acquisition parameters will affect the quality of the data acquired, the time of acquisition, and possibly the success of the experiment. Each parameter should be set with a range wide enough to allow for variation in the tested sample array, but as a smaller range will greatly reduce acquisition time, a balance is required.

\section{Typical equipment being used for automated MALDI and DIOS analyses}

Commercially available instrumentation possess automation packages and more companies are developing this capability. A few companies are also combining their instruments with liquid sample handlers that allow for the rapid deposition of matrix as well as sample.

\section{Automated quantitation studies using MALDI-MS}

Since some biofluids contain contaminants, it is often necessary to perform extractions for purification prior to sample analysis. In such instances, the development of an efficient extraction assay can be very time-consuming. In an effort to create a simpler and more efficient extraction protocol, we have developed a combinatorial extraction method to be used with automated MALDI mass spectrometry, ultimately to improve quantitative clinical analysis of the immunosuppressant drug cyclosporin A (CsA) [15]. This combinatorial-extraction approach was followed by analyses using a MALDI mass spectrometer equipped with automated multi-sampling capabilities to facilitate data collection and analysis of cyclosporin A (Fig. 8). The organic layer extracted from each blood sample was placed on a MALDI sample plate (with a capacity for one hundred samples) and was then loaded into the mass spectrometer and analyzed using a computer-controlled algorithm. Extraction optimization was performed by generating an array of solvent systems, followed by automated analysis to identify successful extractions. The first generation of experiments revealed four binary solvent systems to be effective for cyclosporin extraction (hexane/EtOH, $\mathrm{ACN} / \mathrm{H}_{2} \mathrm{O}, \mathrm{ACN} / \mathrm{MeOH}$, hexane $/ \mathrm{CHCl}_{3}$ ). A new array based on these solvent systems was generated and in a second iteration of experiments, hexane/ $\mathrm{CHCl}_{3}(70: 30)$ was found to provide the most effective and rapid (typically requiring 5 minutes to perform) single-step extraction for cyclosporin and its metabolites. The limits of detection were determined to be $15 \mathrm{ng} / \mathrm{ml}$ in whole blood for both ESI/MS and MALDI-MS and could be used to identify major drug metabolites.

In order to determine the efficiency of our new extraction procedure, it was compared to a previously developed "ether" extraction which typically required an hour to perform [13,14]. Initially, calibration curves were obtained using the "ether" extraction (a plot of the ratio of $\sum$ int. $\left[\mathrm{CsAH}^{+}+\right.$

Table 3

The results obtained from the ESI-MS and MALDI-MS analysis of extracted cyclosporin A from standard blood samples using the $70 / 30$ hexane/ $\mathrm{CHCl}_{3}$ extraction

\begin{tabular}{ccc}
\hline $\begin{array}{c}\text { Standard } \\
\mathrm{ng} / \mathrm{ml}\end{array}$ & $\begin{array}{c}\text { ESI } \\
\mathrm{ng} / \mathrm{ml} \text { (error) }\end{array}$ & $\begin{array}{c}\text { MALDI } \\
\mathrm{ng} / \mathrm{ml}(\text { error) }\end{array}$ \\
\hline 100 & $98(2 \%)$ & $94(6 \%)$ \\
250 & $231(8 \%)$ & $238(5 \%)$ \\
500 & $523(5 \%)$ & $543(9 \%)$ \\
1000 & $1009(1 \%)$ & $961(4 \%)$ \\
1500 & $1404(6 \%)$ & $1514(1 \%)$ \\
\hline
\end{tabular}


$\left.\mathrm{CsANa}^{+}\right] /\left[\mathrm{CsGH}^{+}+\mathrm{CsGNa}^{+}\right]$vs $\mathrm{CsA}$ concentration) with both the ESI and MALDI mass spectrometers (cyclosporin G, CsG, was used as the internal standard). The data exhibited a linear relationship in the concentration range ( 0 to $1500 \mathrm{ng} / \mathrm{ml})$ (Table 3 ) with an excellent correlation coefficient $(\mathrm{R}=0.999)$ for both electrospray and MALDI.

\section{Automated genotyping using MALDI-MS}

Single-nucleotide polymorphisms (SNPs) are point mutations in the genetic material of individuals. These mutations appear with variable frequency between 1 in every 300-1000 nucleotides within the human genome [29,30] making them the most common type of human genetic variation. While many mutations have little functional relevance, some have been linked to particular diseases. Genetic mutations have been implicated in cystic fibrosis, muscular dystrophy, Alzheimer's disease, and many forms of cancer including breast, ovarian and prostate cancer. Because SNPs are defined mutations both in type and locality of the changes, they will provide a rapid method for mapping genetic variability in a population and for determining genetic susceptibility to disease. For this reason, a massive effort has

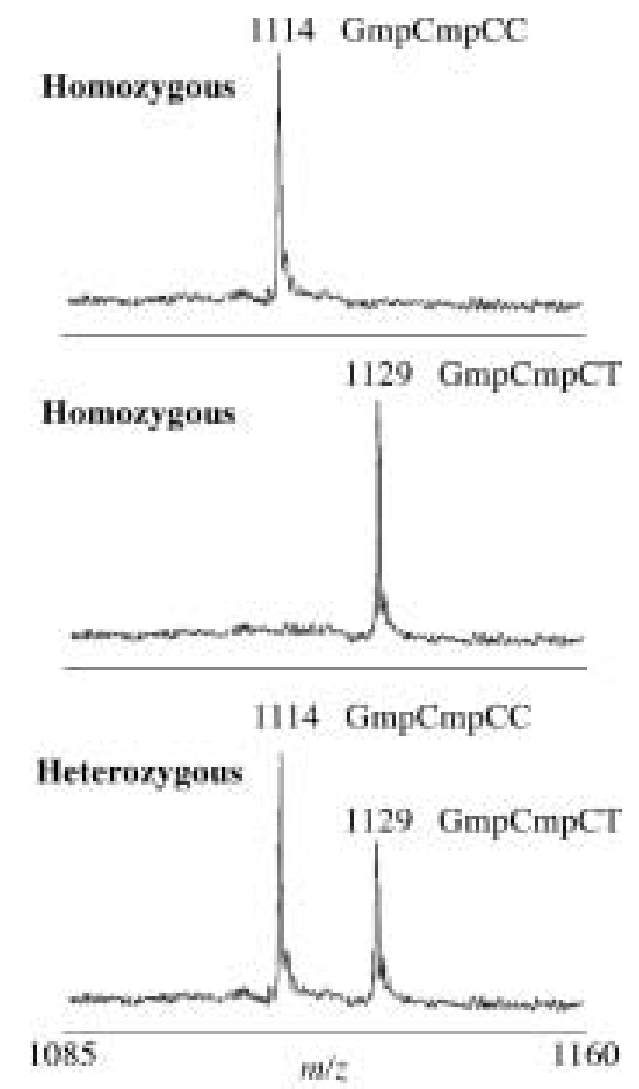

Fig. 9. Representative MALDI-TOF positive ion mass spectra for genotyping of an SNP on the human $\beta-2$ adrenergic receptor using the modified GOOD assay. A 10mer primer for the SNP position of interest was extended with ddTTP and ddCTP. After digestion with phosphodiesterase II, the products have masses of $1114 \mathrm{Da}$ after incorporation of ddCTPs and 1129 Da after incorporation of ddTTPs. The spectra show DNA homozygous for (a) C, (b), T (c) and heterozygous DNA. mp = methylphosphonate. 
been underway to determine rapid high-throughput methods for mapping SNP mutations throughout the genome [31,32].

To enable genome-wide genetic studies using SNPs, dense genetic maps are needed, consisting of at least 1 informative SNP per 30 kilobases $\left(1 \times 10^{5}\right.$ total SNPs). In addition, to determine the clinical relevance of SNPs, comparative studies of the genetic differences between thousands of affected and unaffected individuals are needed. These undertakings require robust, flexible and inexpensive assays and platforms providing a high degree of multiplexing. MALDI-TOF is an important technology to facilitate high-throughput mapping of SNPs, offering speed and high specificity [33]. The time required for a typical MALDI-TOF analysis is very short, less than ten seconds, and the masses detected are based on the absolute mass for the molecules being analyzed. Most importantly, all the steps required for complete analysis can be automated. An example of MALDI-TOF analysis of an SNP on the human $\beta-2$ adrenergic receptor is shown in Fig. 9. SNPs in the human $\beta-2$ adrenergic receptor are linked to a genetic predisposition to hypertension and are also implicated in cardiovascular disease [34].

Common SNP analysis methods currently include the PinPoint assay and the simplified GOOD assay. The PinPoint assay is based on the addition of a single nucleotide to a genotyping primer complementary to the PCR analysis of point mutations, the products of which, short oligonucleotides, are then analyzed by MALDI-MS. This assay supports multiplexing by using mass-resolved primers, as demonstrated in a fivefold multiplex assay for mutations in exon 13 of the BRCAl gene [35], a twelve fold multiplex assay for amplifying loci that have a high degree of heterozygosity amongst healthy individuals [36] and the high throughput genotyping of the human Y chromosome [37]. The simplified GOOD assay is a purification-free, three-step method (PCR, primer extension and phosphodiesterase II digestion) performed in a single tube, immediately followed by MALDI analysis. A chemical modification procedure known as charge-tagging - the conditioning of products to carry either a single excess positive or negative charge-improves the sensitivity 100 -fold and renders the allele-specific products insensitive to impurities such as the preparation buffers in MALDI. For MALDI analysis of SNPs, the reaction mixture can be diluted in acetonitrile [38] and then transferred onto matrix prepared MALDI target plates, such as $\alpha$-cyano-4-hydroxy cinnamic acid methyl ester. Alternative matrix mixtures are 3-hydroxypicolinic acid in a 1:1:2 mixture of water, acetonitrile and $0.1 \mathrm{M}$ ammonium citrate [39] and $10 \mathrm{mg} / \mathrm{ml}$ sinapinic in $2: 1$ water/acetonitrile solution [40].

\section{Characterization of molecules directly from the solid phase}

The MALDI and ESI automation approaches of screening for enzyme inhibitor activity and optimizing extraction conditions, described in this manuscript, represent only two of the many ways in which mass spectrometry can be used in the field of high throughput chemistry. In addition to the development of new quantitative methodologies, the qualitative evaluation of combinatorial libraries is essential for the characterization of active compounds and for monitoring both the progression and efficiency of reaction pathways [1-3]. Because many libraries are synthesized on solid polymeric supports such as resin beads, this chemistry necessitates assays which allow for characterization directly from the solid support $[5,11]$. The following section provides a description of MALDI-MS and photolabile linkers as a means of directly characterizing compounds from the solid phase.

Several reports have shown the utility of mass spectrometry in the characterization of compounds subsequent to their chemical cleavage from solid polymeric supports. Demonstrated here is a new MALDI approach which allows compounds covalently bound to a single polymeric bead ( $\sim 50 \mu \mathrm{m}$ in diameter) 

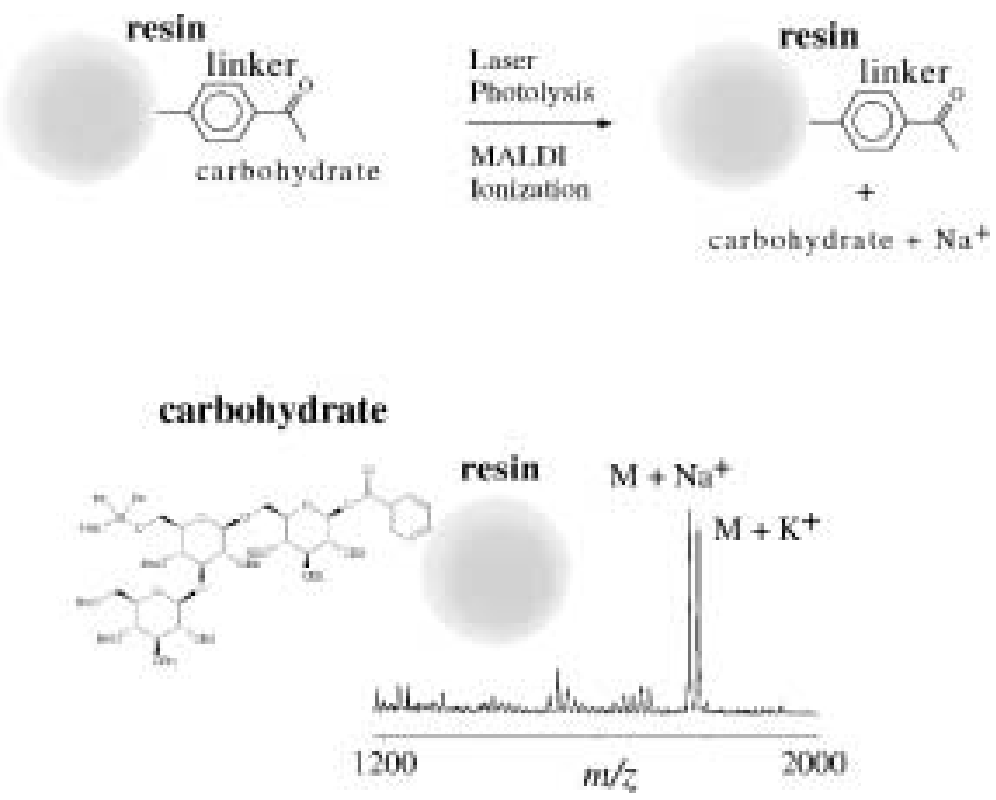

Fig. 10. The MALDI technique facilitates the vaporization and ionization of analyte molecules by irradiating the sample/matrix mixture with a UV laser. (Top) Schematic representation of the products generated upon laser photolysis (by MALDI) of a compound covalently attached to a solid phase resin through a photolabile $\alpha$-methylphenacyl-ester linker. (Bottom) The mass spectrum of the carbohydrate obtained.

or set of beads to be mass analyzed prior to their chemical cleavage from the resin [16]. The scheme, outlined in Fig. 10, permits the characterization of resin-bound analytes in a single step, requiring no pretreatment of the sample to induce cleavage from the support. Therefore, peptides covalently linked to a polymeric support through a photolabile linker can be directly analyzed by MALDI-MS. This procedure is also suitable for monitoring chemical reactions on the solid phase.

The peptide KPAFLKPQFLG was manually synthesized from protected amino acids in a stepwise fashion using in situ neutralization/HBTU activation protocols for Boc chemistry. The synthesis was initiated on Br-Wang resin (obtained from Novabiochem, San Diego, CA) consisting of a brominated $\alpha$-methylphenacyl linker attached to a polystryrene-1\% divinyl benzene support. Prior to mass spectral analysis, resin samples were treated with $20 \%(\mathrm{v} / \mathrm{v})$ piperdine in DMF in order to remove the Fmoc protecting groups, and then washed with $50 / 50(\mathrm{v} / \mathrm{v})$ dichloromethane/methanol.

Our primary goal in this work was to determine if the MALDI experiment could be used to simultaneously promote both photolytic cleavage (from the resin bead) and gas phase ionization of the analyte for subsequent mass spectral analysis. Therefore, $\sim 1 \mathrm{mg}$ of the deprotected peptide resin was suspended in a large solvent volume $(\sim 5 \mathrm{ml})$ in order to sufficiently dilute the residual free peptide in the sample to undetectable levels; resuspension in smaller volumes of solvent $(0.01-1.0 \mathrm{ml})$, led to the detection of free peptide in the MALDI analysis of the supernatant. Presumably, trace amounts of peptide $(<1 \mathrm{nmol}$ or $<1 \%$ of the resin bound material) were present as a result of photolytic cleavage by room light. A $2 \mu \mathrm{l}$ ethanol/bead suspension containing $\sim 50$ beads was then deposited on the MALDI sample plate, prior to the addition of $2 \mu \mathrm{l}$ of a saturated ethanol solution of the matrix, $\alpha$-cyano-4-hydroxycinnamic acid.

Figure 10 shows the mass spectrum of the unprotected, resin-bound compound for which MALDI analysis yielded a characteristic $[\mathrm{M}+\mathrm{Na}]^{+}$signal. Mass spectra obtained from a single bead with one laser pulse (3 nanoseconds in duration) indicated that photolytic cleavage and ionization of material from 


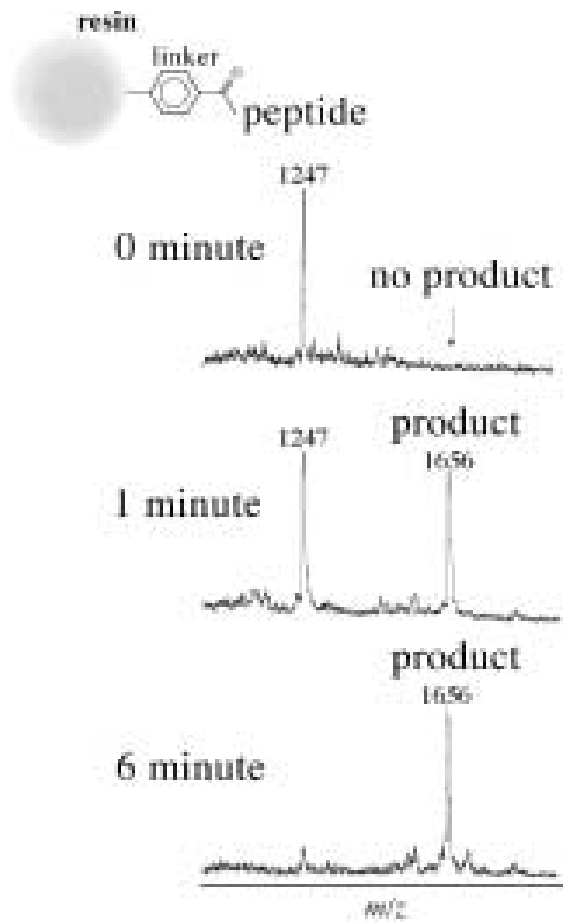

Fig. 11. MALDI mass spectra showing the time course of reaction for the coupling of Boc-Arg(Tos) (MW $=428.5$ Da) to the protected resin-bound peptide of sequence KPAFLKPQFLG. MALDI analysis was performed directly on peptide-resin samples after deprotection of the lysine side chain amino groups. Resin samples were taken after the reaction and allowed to proceed for 0,1 , and 6 minutes.

the bead occurred during the laser pulse, as the resin sample was not exposed to laser light prior to analysis. The rapid, one-step MALDI procedure for the direct analysis of resin-bound molecules described above is ideally suited for studying chemical reactions on the solid phase. As a demonstration of the utility of MALDI in such an analysis, the coupling reaction of a Boc-Arg(Tos) residue to the protected 11 amino acid peptide was monitored as a function of time (Fig. 11). The reaction proceeded quickly and coupling appeared nearly complete in just six minutes.

The direct analysis of resin-bound molecules by MALDI offers several important advantages, including the lack of an additional cleavage step prior to mass analysis (often required by other methods of characterization). MALDI also requires less sample handling and more efficient management since the resin-bound compound can be easily recovered for subsequent manipulations (again, $<$ femtomoles of material is consumed in typical MALDI analyses). The most significant advantage is that it can be used to monitor chemical reactions on the solid phase in real time, in much the same way that thin-layer chromatography is used to monitor reactions in solution. Furthermore, all analytes amenable to MALDI ionization should prove suitable for routine analysis by this procedure.

\section{Conclusion/summary}

The rapid growth of high throughput chemistry has created a need for faster, more accurate, and more sensitive analytical techniques capable of large-scale screening. Numerous improvements in speed, sen- 
sitivity and accuracy, together with innovations in both automation and quantitation, place mass spectrometry among the most powerful analytical techniques available today. There are now many analyzer options for high throughput analysis of compounds as well as multiple ionization techniques that have a much greater range of compatible compounds than were available even five years ago. Quantitative ESI-MS has been shown to be an effective assay for the identification of inhibitor activity in a combinatorial library, namely potent nucleotide inhibitors of a galactosyltransferase. Clearly, this approach can be applied to a variety of different reaction systems. A combinatorial extraction method in conjunction with an automated MALDI mass spectrometric procedure was also used to optimize the clinical analysis of the immunosuppressant drug CsA from whole blood. MALDI-based assays are versatile tools for the high-throughput genotyping of SNPs and other point mutations in human DNA. In addition, MALDI was shown to be an effective method for the direct analysis of resin-bound compounds (without chemical cleavage from the resin), an ideal technique for the identification/characterization of combinatorial compounds synthesized on solid supports. Furthermore, DIOS has recently been developed as an effective small molecule and proteomics tool and offers the potential of high throughput analysis of a wide variety of compounds. Overall, the strength of mass spectrometry lies in such versatility, making it a powerful analytical technique with which to characterize the diversity of compounds found in modern high throughput chemistry.

\section{References}

[1] R.S. Youngquist, G.R. Fuentes, M.P. Lacey and T. Keough, Generation and screening of combinatorial peptide libraries designed for rapid sequencing by mass spectrometry, J. Am. Chem. Soc. 117 (1995), 3900-3906.

[2] J.A. Boutin, P. Hennig, P.H. Lambert, S. Bertin, L. Petit, J.-P. Mahieu, B. Serkiz, J.-P. Volland and J.-L. Fáuchere, Combinatorial peptide libraries - robotic synthesis and analysis by NMR, mass spectrometry, tandem mass spectrometry and high-performance capillary electrophoresis techniques, Anal. Biochem. 234 (1996), 126-141.

[3] J. Gao, X. Cheng, R. Chen, G.B. Sigal, J.E. Bruce, B.L. Schwartz, S.A. Hofstadler, G.A. Anderson, R.D. Smith and G.M. Whitesides, Screening derivatized peptide libraries for tight binding inhibitors to carbonic anhydrase II by electrospray ionization-mass spectrometry, J. Med. Chem. 39 (1996), 1949-1955.

[4] G. Hegy, E. Gorlach, R. Richmond and F. Bitsch, High throughput electrospray mass spectrometry of combinatorial chemistry racks with automated contamination surveillance and results reporting, Rapid Commun. Mass Spectrom. 10 (1996), 1894-1900.

[5] C.L. Brummel, J.C. Vickerman, S.A. Carr, M.E. Hemling, G.D. Roberts, W. Johnson, J. Weinstock, D. Gaitonopoulos, S.J. Benkovic and N. Winograd, Evaluation of mass spectrometric methods applicable to the direct analysis of non-peptide bead-bound combinatorial libraries, Anal. Chem. 68 (1996), 237-242.

[6] T.A. Berger, K. Fogelman, T. Staats, P. Bente, I. Crocket, W. Farrell and M. Osonubi, The development of a semi-preparatory scale supercritical-fluid chromatograph for high-throughput purification of 'combi-chem' libraries, J. Biochem. Biophys. Methods 43(1-3) (2000), 87-111.

[7] J.D. Henion, A.V. Nlordehai and J.Y. Cai, Quantitative capillary electrophoresis ion spray mass spectrometry on a benchtop ion for the determination of isoquinoline alkaloids, Anal. Chem. 66 (1994), 2103-2109.

[8] E.D. Lee, W. Muck, J.D. Henion and T.R. Covey, Real-time reaction monitoring by continuous-introduction ion-spray tandem mass spectrometry, J. Am. Chem. Soc. 111 (1989), 4600-4604.

[9] S. Takayama, R. Martin, J. Wu, K. Laslo, L. Qiao, G. Siuzdak and C.-H. Wong, Chemoenzymatic preparation of novel cyclic imine sugars and rapid biological activity evaluation using electrospray mass spectrometry and kinetic analysis, J. Am. Chem. Soc. 119 (1997), 8146-8151.

[10] J. Wu, T. Shuichi, C.-H. Wong and G. Siuzdak, Quantitative electrospray mass spectrometry for the rapid assay of enzyme inhibitors, Chemistry and Biology 4 (1997), 653-657.

[11] B.J. Egner, G.J. Langley and M. Bradley, Solid phase chemistry - direct monitoring by matrix-assisted laser desorption ionization time of flight mass spectrometry - A tool for combinatorial chemistry, J. Org. Chem. 60 (1995), 2652-2653.

[12] D. Rideout, A. Bustamante and G. Siuzdak, Cationic drug analysis using matrix-assisted laser desorption ionization mass spectrometry - application to influx kinetics, multidrug resistance and intracellular chemical change, Proc. Nat. Acad. Sci. USA 90 (1993), $10226-10229$. 
[13] D.C. Muddiman, A.I. Gusev, A. Proctor, D.M. Hercules, R. Venkataramanan and W. Diven, Quantitative measurement of cyclosporin A in blood by time-of-flight mass spectrometry, Analyt. Chem. 66 (1994), 2362-2368.

[14] D.C. Muddiman, A.I. Gusev, K.S. Langner, A. Proctor, D.M. Hercules, P. Tata, R. Venkataramanan and W. Diven, Simultaneous quantification of cyclosporin A and its major metabolites by TOF SIMS and MALDI MS utilizing data analysis techniques: comparison with HPLC, J. Mass Spec. 30 (1995), 1469-1479.

[15] J. Wu, K. Chatman, K. Harris and G. Siuzdak, An automated MALDI mass spectrometry approach for optimizing cyclosporin extraction and quantitation, Anal. Chem. 69 (1997), 3767-3771.

[16] M.C. Fitzgerald, K. Harris, C.G. Shevlin and G. Siuzdak, Direct characterization of solid phase resin-bound molecules by mass spectrometry, Bioorganic and Medicinal Chemistry Letters 6 (1996), 979-982.

[17] G. Siuzdak, Mass Spectrometry for Biotechnology, Academic Press, San Diego, 1996.

[18] M.C. Ventura, W.P. Farrell, C.A. Aurigemma and M.J. Greig, Packed column supercritical fluid chromatography/mass spectrometry for high-throughput analysis. Part 2, Anal. Chem. 71 (1999), 4223-4231.

[19] S.H. Hoke, J.A. Tomlinson II, R.D. Bolden, K.L. Morand, J.D. Pinkston and K.R. Wehmeyer, Increasing bioanalytical throughput using pcSFC-MS/MS: 10 minutes per 96-well plate, Anal. Chem. 73 (2001), 3083-3088.

[20] J.A. Loo, D.E. DeJohn, P. Du, T.I. Stevenson and R.R. Ogorzalek-Loo, Application of mass spectrometry for target identification and characterization, Med. Res. Rev. 19(4) (1999), 307-319.

[21] H. Lee, T.J. Griffin, S.P. Gygi, B. Rist and R. Aebersold, Development of a multiplexed microcapillary liquid chromatography system for high-throughput proteome analysis, Analyt. Chem. 74(17) (2002), 4353-4360.

[22] S.M. Singh, A.A. Zada, W. Hiddemann, D.G. Tenen, V.A. Reddy and G. Behre, Proteomic analysis of transcription factor interactions in myeloid stem cell development and leukaemia, Expert. Opin. Ther. Targets 6(4) (2002), 491-495.

[23] D.B. Robb, T.R. Covey and A.P. Bruins, Atmospheric pressure photoionization: an ionization method for liquid chromatography-mass spectrometry, Anal. Chem. 72(15) (2000), 3653-3659.

[24] J.A. Syage, M.D. Evans and K.A. Hanold, Photoionization mass spectrometry, American Laboratory (2000), 24-29.

[25] J.A. Syage and M.D. Evans, Photoionization mass spectrometry - a powerful new tool for drug discovery, Spectroscopy 16(11) (2001), 15-22.

[26] M.K. Bayliss, D. Little, D.N. Mallett and R.S. Plumb, Parallel ultra-high flow rate liquid chromatography with mass spectrometric detection using a multiplex electrospray source for direct, sensitive determination of pharmaceuticals in plasma at extremely high throughput, Rapid Commun. Mass Spectrom. 14(21) (2000), 2039-2045.

[27] D.G. Morgan, M.S. Villeneuve and L.W. Frick, Use of basic additives with SFC/MS. Poster presented at the 10th International Symposium and Exhibition on Supercritical Fluid Chromatography, Extraction and Processing, Myrtle Beach, SC, USA, August 19-22, 2000.

[28] M.J. Greig, submitted.

[29] J.C. Venter et al., The sequence of the human genome, Science 291 (2001), 1304-1351

[30] T.J. Griffin and L.M. Smith, Single-nucleotide polymorphism analysis by MALDI-TOF mass spectrometry, TIBTECH 18 (2000), 77-84.

[31] K. Tang, D.-J. Fu, D. Julien, A. Braun, C.R. Cantor and H. Koster, Chip-based genotyping by mass spectrometry, Proc. Natl. Acad. Sci. USA 96 (1999), 10 016-10 020.

[32] K.H. Buetow, M. Edmonson, R. MacDonald et al., High-throughput development and characterization of a genome wide collection of gene-based single nucleotide polymorphism markers by chip-based matrix-assisted laser desorption/ionization time-of-flight mass spectrometry, Proc. Natl. Acad. Sci. USA 98 (2001), 581-584.

[33] W. Pusch, J.H. Wurmbach, H. Thiele and M. Kostrzewa, MALDI-TOF mass spectrometry-based SNP genotyping, Pharmacogenomics 3(4) (2002), 537-548.

[34] A. Busjahn, G.-H. Li, H.-D. Faulhaber, M. Rosenthal, A. Becker, E. Jeschke, H. Schuster, B. Timmerman, M.R. Hoehe and F.C. Luft, $\beta$-Adrenergic receptor gene variations, blood pressure and heart size in normal twins, Hypertension 35 (2000), 555-560.

[35] L.A. Haff and I.P. Smirnov, Multiplex genotyping of PCR products with MassTag-labeled primers, Nucleic Acids Res. 25(18) (1997), 3749-3750.

[36] P. Ross, L. Hall, I. Smirnov and L. Haff, High level multiplex genotyping by MALDI-TOF mass spectrometry, Nat. Biotechnol. 16(13) (1998), 1347-1351.

[37] S. Paracchini, B. Arredi, R. Chalk and C. Tyler-Smith, Hierarchical high-throughput SNP genotyping of the human Y chromosome using MALDI-TOF mass spectrometry, Nucleic Acids Res. 30(6) (2002), e27.

[38] S. Sauer, D.H. Gelfand, F. Boussicault, K. Bauer, F. Reichert and I.G. Gut, Facile method for automated genotyping of single nucleotide polymorphisms by mass spectrometry, Nucleic Acids Res. 30(5) (2002), e22.

[39] X. Chen, Z. Fei, L.M. Smith, E.M. Bradbury and V. Majidi, Stable-isotope-assisted MALDI-TOF mass spectrometry for accurate determination of nucleotide compositions of PCR products, Anal. Chem. 71(15) (1999), 3118-3125.

[40] P.L. Ross, K. Lee and P. Belgrader, Discrimination of single-nucleotide polymorphisms in human DNA using peptide nucleic acid probes detected by MALDI-TOF mass spectrometry, Analyt. Chem. 69(20) (1997), 4197-4202. 


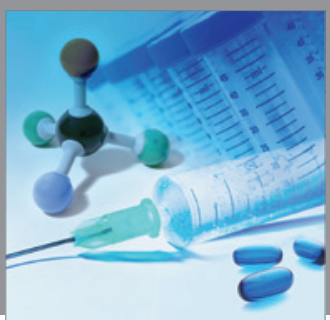

International Journal of

Medicinal Chemistry

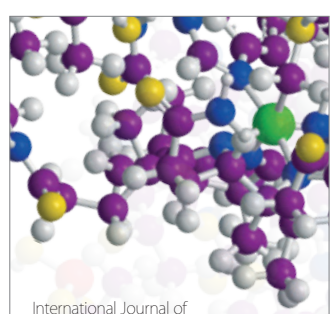

Carbohydrate Chemistry

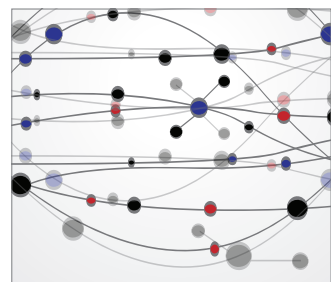

The Scientific World Journal
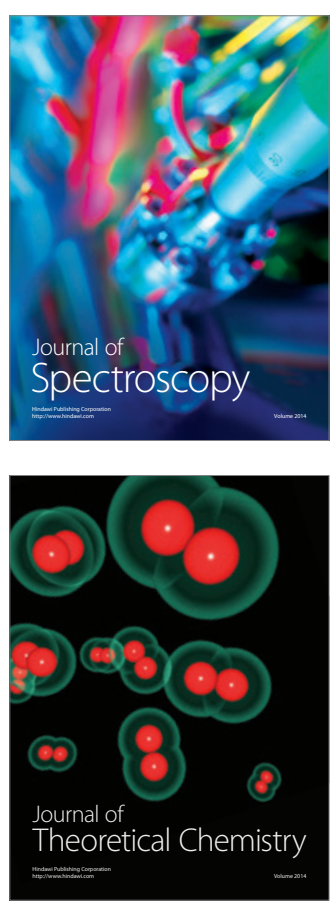
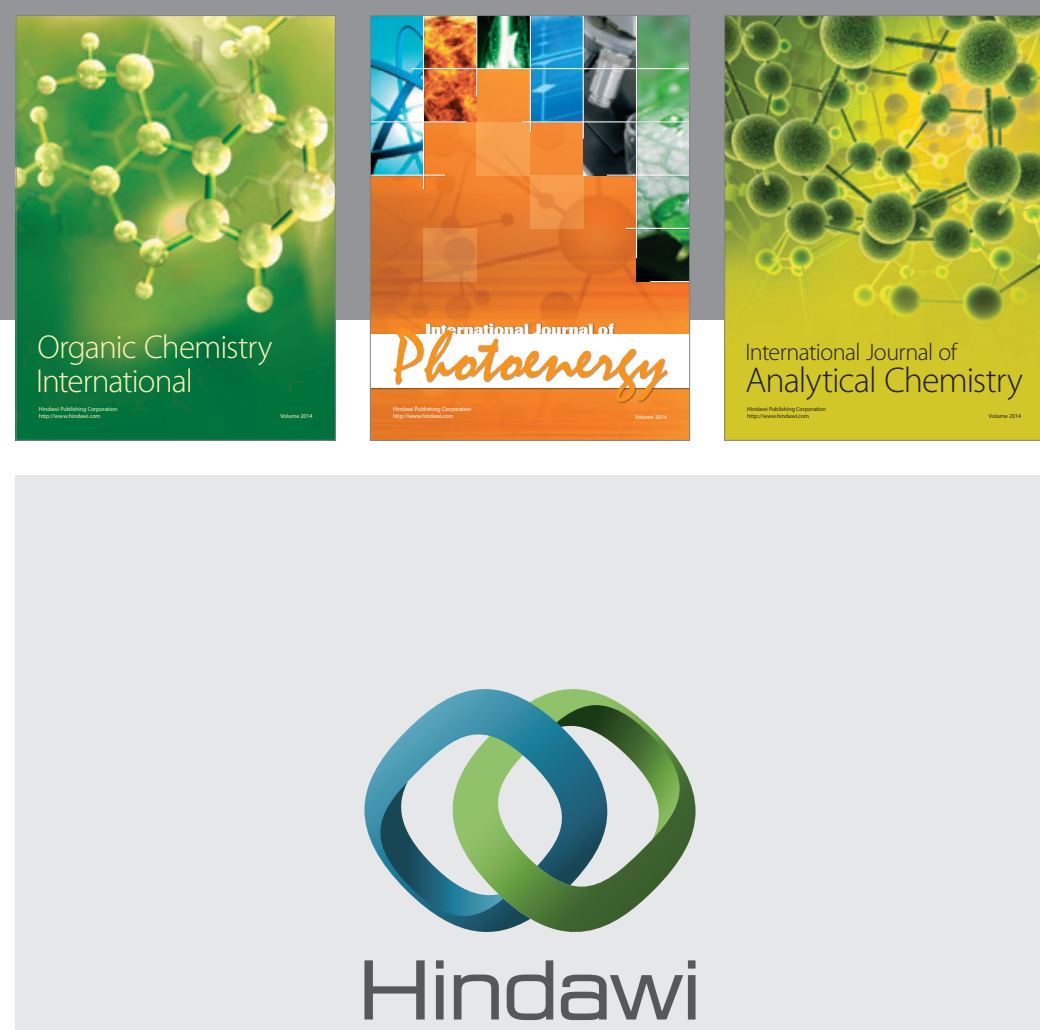

Submit your manuscripts at

http://www.hindawi.com
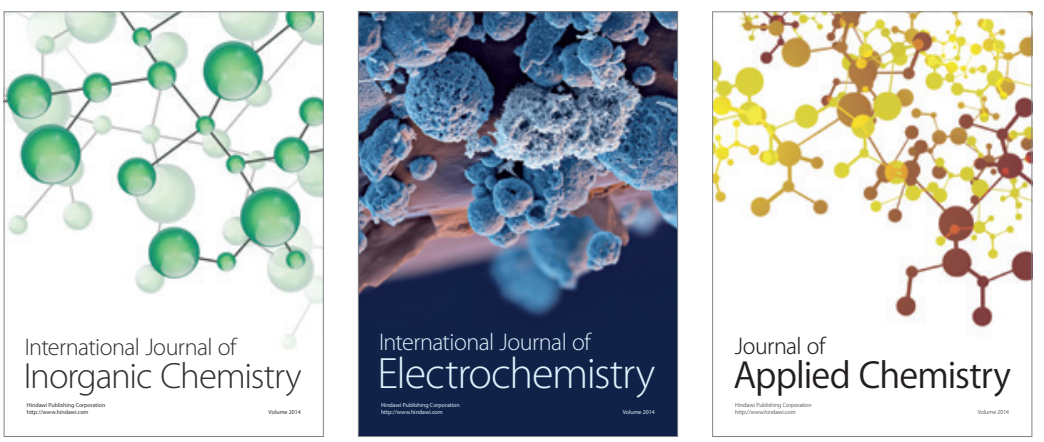

Journal of

Applied Chemistry
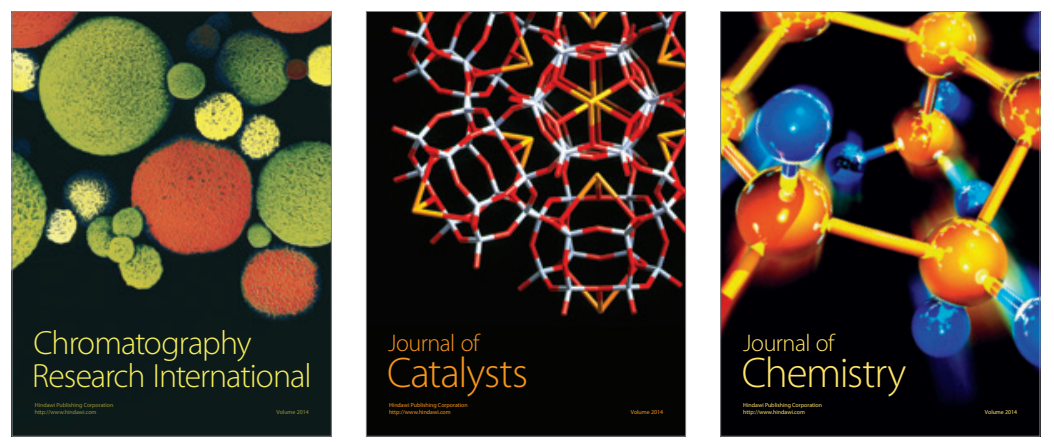
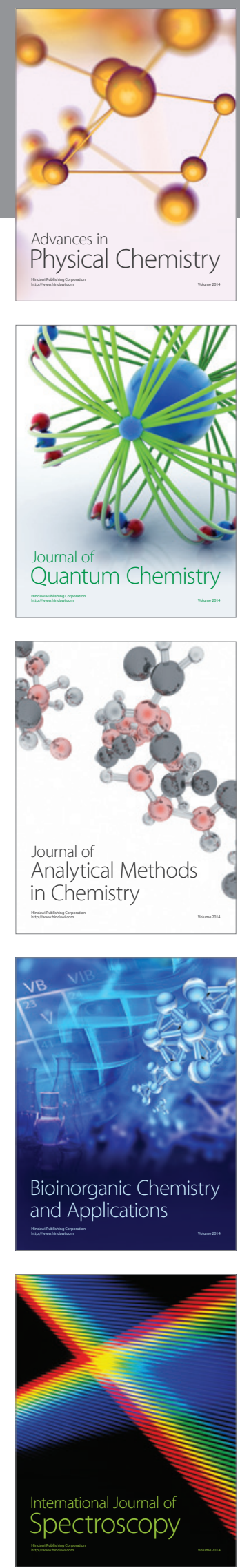